\title{
Platelet-derived growth factors induced lymphangiogenesis: evidence, unanswered questions and upcoming challenges
}

\author{
Andreea Adriana Jitariu, Anca Maria Cimpean, Nilima Rajpal Kundnani, Marius Raica
}

Victor Babes University of Medicine and Pharmacy, Timisoara, Romania

Submitted: 28 October 2013

Accepted: 4 December 2013

Arch Med Sci 2015; 11, 1: 57-66

DOI: 10.5114/aoms.2015.49217

Copyright $@ 2015$ Termedia \& Banach

\section{Abstract}

Crosstalk between angiogenesis and lymphangiogenesis in embryonic development continues during postnatal life and has specific mechanisms involving factors that initiate activation of the intracellular cascade for their specific receptors. Platelet-derived growth factors (PDGFs) and their corresponding receptors (PDGFRs) are known as important regulators of blood vessel development in both normal and pathologic angiogenesis. Despite some recent papers which reported a potential role of the PDGF/ PDGFR axis in lymphatic spread of tumor cells, a few papers have suggested the potential role of PDGFs in tumor lymphangiogenesis development. The present paper summarizes the potential lymphangiogenic role of the PDGF/ PDGFR axis, underlying upcoming challenges in the field.

Key words: lymphangiogenesis, platelet-derived growth factor receptor, platelet-derived growth factor, tumor microenvironment.

\section{PDGF/PDGFR system: structure and signaling pathways}

The platelet-derived growth factor (PDGF) family was identified in platelets and in serum as fibroblast, smooth muscle cell and glia culture cell mitogen factors [1]. Four members of this family have been evidenced, noted as A, B, C and D, the latter two types being recently discovered. The active form of the PDGF presents as a homodimer or a heterodimer; in the PDGF family four homodimers (PDGF-AA, PDGF-BB, PDGF-CC and PDGF-DD) and only one heterodimeric form (PDGF-AB) are recognized. The PDGF dimers are assembled within the cell [2]. Configurations of certain types have the assembly of isotypes $A$ and $B$ accomplished without a specific orientation $[3,4]$. This is the reason behind a great deal of structural similarities between the $A$ and $B$ type of PDGF receptors [5]. Certain cells may therefore produce PDGF-AA or PDGF-BB, if any of the genes that encode PDGF synthesis are translated in a singular fashion [5]. In case of simultaneous transcription of both the genes, the result will be a mixture that will include PDGF-AA, PDGF-BB and PDGF-AB [4]. In addition to this, noticing the primary sequence of the polypeptide chain, it is seems that PDGF-C and -D are not strongly related to PDGF-A and $-B$, which explains the impossibility to form heterodimers with the two isotypes [5]. The possibility to form heterodimers between types $C$ and $D$ remains at the level of a hypothesis [1]. PDGF-C and PDGF-D possess an

\author{
Corresponding author: \\ Assoc. Prof. Anca Maria \\ Cimpean MD, PhD \\ Victor Babes University \\ of Medicine and Pharmacy \\ Piata Eftimie Murgu 2 \\ 300041 Timisoara, Romania \\ Fax: 40720060955 \\ E-mail: ancacimpean1972@ \\ yahoo.com
}


$\mathrm{N}$-terminal domain named the CUB domain with a function unknown for the time being. It appears that during the growth factor secretion through proteolytic processing, the CUB domains are not required.

There are two binding receptors for PDGFs, named PDGFR $\alpha$ and PDGFR $\beta$. PDGF-dependent receptor dimerization is at first needed for signal auto-phosphorylation and transmission [1]. PDGFR $\beta$ presents high affinity for PDGF-B and PDGF-D [6]. PDGFR $\alpha$ has high specificity for PDGF-A [7] and PDGF-C [8] but also for PDGF-B (the receptor affinity being greater for PDGF-A than for PDGF-B). The gene expression for the two receptor types might be in an individual manner or together in a single cell [1]. The PDGF signaling receptor structure unity may be PDGFR $\alpha \alpha$, PDGFR $\beta \beta$ or PDGFR $\alpha \beta$. This highly depends on the gene expression for the PDGF receptor pattern, as well as on the viability of different family dimers. The studies carried on PDGF receptors have been based especially on the $\beta$ form. Through the intracellular residues of phosphotyrosine, receptor dimers come in contact with a certain number of $\mathrm{SH} 2$ domains containing molecules that ensure the bond with a number of signaling pathways $[1,9]$. In the cytoplasmic matrix the protein categories which bind PDGFR $\beta$, namely PLC- $\gamma$, PI3K [10], Ras, GAP, SHP2 phosphatase and the family of Src kinases, are included [1, $9,11]$. These pathways seem to be in connection with genes that activate immediately [9]. The phospho-tyrosine residues within PDGFR $\beta$ molecules are implicated in binding and activating a certain signaling pathway. The differences that appear in receptor binding are found in the case of the Ras GAP pathway, which binds only PDGFR $\beta$, but not PDGFR $\alpha$, and in the case of the Crk adaptor protein family, which binds only PDGFR $\alpha$ [1]. Receptors have different roles during development [12], an aspect that is mostly associated with pattern expression. It is unknown whether the role played by the $\beta$ type (in the case of its absence) could be totally compensated by the presence of the $\alpha$ form.

The PDGF stimulates cells in a specific manner, through binding to the cell surface receptors, and that determine DNA synthesis and cell proliferation [7, 13]. The PDGFR rather than the factor itself may possess activities or sites needed for proliferation response initiation in target cells. There is evidence that if the cells are preincubated with PDGF, the activity of PDGFR is much reduced. The number of binding sites decreases to $10 \%$ after the cell is pre-incubated with $200 \mathrm{ng} / \mathrm{ml}$ of PDGF. Dimer BB of the PDGF family is expressed by endothelial cells (ECS) and is implicated in signal induction through binding only to PDGFR $\beta$ expressed by perivascular cells and ECs [14]. However, numerous experimental studies have shown that
PDGF is expressed by ECs within a temporal-spatial pattern. The PDGF-B endothelial expression is at first largely spread, but afterwards it becomes restricted to ECs from the sprouting tips [14]. It seems that the tip cell maintains a tight contact with pericytes. This contact is a key element that facilitates PDGF-B release.

Pericyte recruitment is one of the essential levels which concerns angiogenesis induced by PDGF family members, and a phenomenon that completely depends on the PDGF-B/PDGFR $\beta$ system signaling pathway [15]. This affinity might be an explanation for almost complete loss of pericytes in the nervous system (and also in other organs) in the absence of the B dimer or PDGFR $\beta$. Also in angiogenesis, the function of PDGF- $B$ is expressed much better in the arterial endothelium than in the venous one [14].

\section{Implication of PDGFs and PDGFRs in pathological lymphangiogenesis and lymph node metastasis}

The PDGF-BB is a direct lymphangiogenic factor that stimulates lymphatic metastasis [16-19]. The PDGFR $\alpha$ as well as PDGFR $\beta$ expressed in an isolated manner in lymphatic endothelial cells (LECs) and all three PDGF prototypes (AA, BB and CC) are capable of inducing lymphangiogenesis [20]. In addition, the growth factors from the PDGF family, as well as the hepatocyte growth factor, have a stimulating effect on the vascular endothelial growth factor family members (with proangiogenic and lymphangiogenic effects) [21-24]. It seems that within the vascular endothelial growth factor (VEGF) family, dimer $A$ has in itself a stimulating effect on PDGF-B expression in ECs $[25,26]$, while FGF-2 stimulates the perivascular expression of PDGFR $\beta$.

Co-stimulation of PDGFs through both VEGF and fibroblast growth factor (FGF) actions determines the recruitment of perivascular cells in vitro as well as determining the formation of a functional vasculature in vivo. In order to accomplish these two effects of PDGF, the preservation of the PDGF peri-endothelial gradient is of a great importance. The two effects of PDGF are suppressed by neutralizing antibody action as well as by PDGF of exogenous origin [14]. In vitro, PDGF-BB stimulates MAP-kinase activity as well as the motility of isolated LECs [18]. In vivo, this factor induces lymph vessel growth. PDGF-BB expression in murine fibrosarcoma induces tumor lymphangiogenesis, leading to lymph node metastasis [18]. Tumor cells have the capacity to induce blood vessel and lymphatic vessel formation for the tumor itself.

Besides PDGF family members, of great importance in this matter is the intervention of the vascular endothelial growth factor (VEGF-C and VEGF-D), 
which interacts with a lymphatic endothelial receptor VEGFR-3. As well as in the case of PDGF-B, both VEGF-C and VEGF-D are overexpressed during lymphatic metastasis in several cancer types including pancreatic and gastric cancer [27, 28].

In recent studies performed in order to evidence the new mechanisms regarding pathological lymphangiogenesis, it has been observed that PDGF-BB expression in primary transplanted murine sarcomas leads to lymph node metastasis in the mouse model [29]. Of all PDGF family members, it seems that only PDGF-BB is capable of activating both $\alpha$ and $\beta$ receptors.

In order to experimentally demonstrate the direct implication of PDGF-BB in tumor lymphangiogenesis and lymph node metastasis, a murine lymphangiogenic model has been used. By using a laboratory lymphangiogenic corneal mouse model together with confocal microscopy, it has been demonstrated that PDGF-BB is capable of inducing lymphangiogenesis in the mouse cornea. In normal conditions, the corneal tissue does not possess blood or lymphatic vessels, but after the implantation of the growth factor, in about 2 weeks the corneal vasculature was well developed. The inclusion of homodimer B determines the development of new lymphatic vessels but with a diameter above the average. New blood vessels started from pre-existing vessels located in the limbus [29]. It was demonstrated that PDGF-AA as well as PDGF-AB presents similarities from this point of view with PDGF-BB, although with some differences. It seems that unlike PDGF$B B, P D G F-A A$ determined the formation of a lesser number of lymphatic vessels, while the other member of the family induced the formation of a similar number as PDGF-BB. The topic of discussion would be, in this case, the importance of the $\beta$ receptor considering that both PDGF-AB and PDGF-BB (but not PDGF-AA), play the ligand role in this case. Therefore, PDGF-BB is the factor with a higher lymphangiogenic potential compared to the rest of the family members. The phenomenon of PDGF-BB-induced lymphatic vessel formation is based on a remodeling process.

The direct role of PDGF-BB in lymphangiogenesis sustains the fact that its action does not depend on the VEGF-C/-D/VEGFR-3 pathway. Nor does it depend on the levels of angiopoietin 2, which are not affected by cell stimulation with PDGF-BB cells that express PDGFR $[29,30]$.

The pathway through PDGFs acts in order to induce lymphangiogenesis depending on various intracellular signaling components, common for PDGF-BB and PDGF-AA but also for VEGF-C [4, 20].

The Prox-1 gene is responsible for the lack of venous vessel conversion to a lymphatic vessel; therefore embryos will present a lack of lymphatic vessel structure [31-33]. There is no connection between Prox-1 gene expression and PDGF-BB level, so overexpression of the gene does not induce high levels of PDGF-BB. Therefore, the manner through which PDGF-BB acts for lymphatic vessel development and differentiation uses other pathways than those activated by Prox-1. Despite all these aspects, some authors support a different hypothesis regarding the relationship between Prox-1 and PDGF-BB. Initially, the Prox-1 gene induces lymphatic vessel development, starting from the venous ECs. Some papers assume the existence of independent control of the Prox-1 gene, which regulates lymphatic vessel growth, a development that probably is led by PDGF system activity, later. All tumor types were able to induce lymphatic vessel development, but only PDGF-BB positive tumors have intratumor lymphatic vessels, which infiltrate deeper in the tumor tissue and may facilitate lymphatic metastasis through various mechanisms [19]. Therefore, the main functional implications of PDGF-BB as a direct endogenous pro-lymphangiogenic factor but also as a lymphatic metastasis promoter are on one hand the direct stimuli of tumor cell growth and on the other hand inducers of angiogenesis and lymphangiogenesis.

Recent studies have suggested the role of PDGF-A in tumor invasion, lymph node metastasis and poor prognosis in different types of malignancies. Donnem et al. [34] found for the first time in 2010 a significant association between PDGF-A expression and lymphatic spread in nonsmall cell lung carcinoma, together with co-expression of PDGF-B and VEGFR-3. This first direct evidence about PDGF-A involvement in lymphangiogenesis and lymphatic metastasis was strongly supported by recently published papers about PDGF-A and PDGFR $\alpha$ involvement as predictors of renal tumors. The PDGFR $\alpha$ is constitutively expressed in proximal and distal convoluted tubules and collecting ducts of normal renal parenchyma, being also positive in clear cell renal cell carcinoma and papillary renal cell carcinoma [35]. Also, limited data are available about the role of the PDGF-A/PDGFR $\alpha$ axis in nephroblastomas [36]. Together with PDGF-BB induction of lymphangiogenesis in the tumor microenvironment, PDGF-AA is also involved in tumor metastasis induction and progression, being an important factor in the recruitment of metastatic cells and their commitment through a favorite metastatic site. Metastatic preference of breast and prostate cancer cells for bone is explained by PDGF-AA secretion from osteoclasts and osteoblasts followed by recruitment of highly metastatic PDGFR $\alpha$ positive variants of tumor cells [37-40].

Scattered and controversial data about the role of PDGF-AB in tumor lymphatic vessel development are available at this moment. It seems that 
PDGF-AB has a potential role in tumor lymphangiogenesis by acting through cancer-associated fibroblast and tumor-associated macrophage recruitment followed by activation of both PDGFR $\alpha$ and PDGFR $\beta$ through an inflammatory mediated mechanism [41, 42].

Certified or potential roles of PDGF family members and their known (for PDGF BB/PDGFR $\beta$ ) and still hypothetical (for other PDGF family members) mechanisms of action are summarized in Figure 1.

\section{The role of PDGF-B and PDGFR $\beta$ in human gastric carcinoma lymphangiogenesis}

Functional implications of the PDGF/PDGFR axis in pathological lymphangiogenesis concern human gastric carcinoma. The PDGF-B and PDGFR $\beta$ RNA expression was found significantly higher in the case of patients who presented lymphatic metastasis as well as in the case of patients with diffuse human gastric carcinoma (mainly intestinal type gastric carcinoma) [43]. The PDGF plays a direct role not only in tumor angiogenesis but also in lymphangiogenesis. The PDGF stimulates tumor growth by tumor cell growth induction and is implicated in the stimulation of angiogenesis, and pericyte recruitment. Apart from this, it successfully influences the transendothelial transport of some chemotherapeutic agents in a paracrine manner [43]. After the quantitative mRNA analysis for PDGF-B and PDGFR $\beta$ by means of real-time $P C R$, it can be concluded that patients with positive lymph node metastasis had significantly higher levels of PDGF-B/PDGFR $\beta$ than those who presented negative lymph nodes. In the case of diffuse human gastric carcinoma, it had a higher probability of lymphatic metastasis, this being a basic characteristic that differentiates it from other types of gastric tumors and tumors that affect the digestive system in general. Therefore, patients with such a type of gastric tumor had much higher levels of PDGFs. Hence, it is possible to state that there is an important association between tumor severity and PDGF levels.

\section{PDGF-A, tumor lymphangiogenesis and esophageal carcinoma}

In squamous cell carcinoma of the esophagus, the exact role played by regulating growth factors still remains unknown. A significant increase of lymphatic vessel density in the case of VEGF-A and VEGF-C was observed $[44,45]$. In addition to this, high expression of mRNA for VEGF-A, VEGF-C and PDGF-A is observed. Both the density of lymphatic vessels and that of blood vessels were significantly higher.

The PDGFs induce the growth of the tumor through direct stimulation of cell growth from different types of tumors. Experimental studies on angiogenesis and lymphangiogenesis were based especially on evidencing the functional implications of the vascular endothelial growth factor. In contrast, as per the recent studies, it is evident that PDGFs are a class of potential angiogenic factors. Particularly, the angiogenic activity of PDGFs is stimulated by the presence of other proangiogenic factors [14].

\section{Breast cancer, PDGFs and PDGFRs}

Known and extensively studied as promoters of bone marrow metastases in breast cancer [46-49], PDGFs and corresponding PDGFRs are considered, currently, as potential targets for antitumor and anti-metastatic drugs rather than antiangiogenic or anti-lymphangiogenic targets. Indirect evidence about the role of PDGFs/PDGFRs in breast cancer lymphangiogenesis and breast cancer cell spreading was found in the literature. The PDGF-D is highly expressed in human breast cancer and facilitates tumor growth and lymph node metastasis, making it a potential target in breast cancer. At the same time, PDGF-D increases drug delivery and hence improves the efficacy of chemotherapy through vessel normalization [50, 51]. Some studies have reported a correlation between nodal metastases, peritumoral and intratumoral lymphatic microvessel density in breast cancer, but direct evidence about the PDGF/PDGFR axis in lymphangiogenic onset and support in breast malignancies has not been provided. Recently, Schito et al. reported that human LECs start to proliferate and migrate when they are co/cultured with breast cancer cells expressing HIF- $1 \alpha$ and PDGF-B and demonstrated colocalization of HIF- $1 \alpha$ and PDGF-B, which were correlated with lymphatic vessel area and histological grade by immunohistochemical analysis of paraffin-embedded human breast cancer specimens [52]. These data are supplemented by Semenza (2013), who described the cancer-stromal cell interactions mediated by hypoxia-inducible factors able to promote angiogenesis, lymphangiogenesis, and metastasis by PDGFR activation together with other stromal factors [53]. Limited data support the PDGF-B/PDGFR $\beta$ axis as a tumor promoter from in situ carcinoma to the invasive stage of breast cancer, but none have concerned its involvement in the early steps of lymphangiogenesis [54]. Our preliminary immunohistochemical studies showed high PDGF-B, PDGFR $\alpha$ and PDGFR $\beta$ expression during progression from in situ carcinoma to invasive, metastatic breast cancer in both tumor cells and surrounding lymphatic vessels (Figure 2).

No data about PDGF/PDGFR involvement in the lymphangiogenic process from molecular types of breast cancer are available now. 


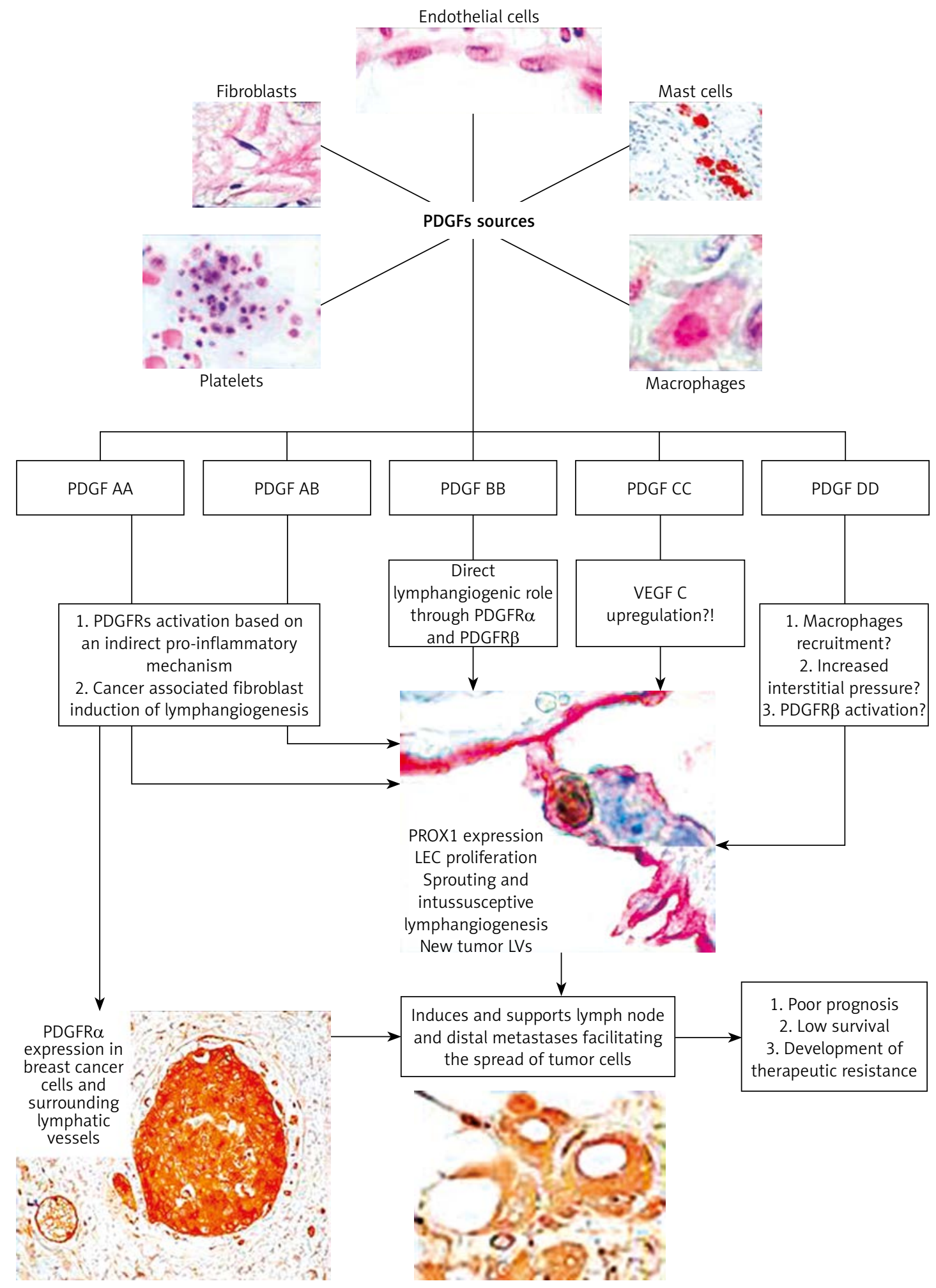

Figure 1. PDGFs' cellular sources, their isoforms and known (for PDGF-B, continuous arrow) or potential (for other forms, marked with dotted arrow) mechanism of action for inducing lymphangiogenesis in tumor conditions with effects on tumor cells and metastasis development and progression

\section{Implications of PDGFs in lymphatic vessel remodeling}

The basis of lymphatic vessel remodeling remains incompletely elucidated. In the context of the role of PDGF in vessel development, studies have revealed that PDGF signals determine the branching of the vasculature directly from the coelomic vessels [55]. Considering the experiments that have been performed on culture cells, it has 

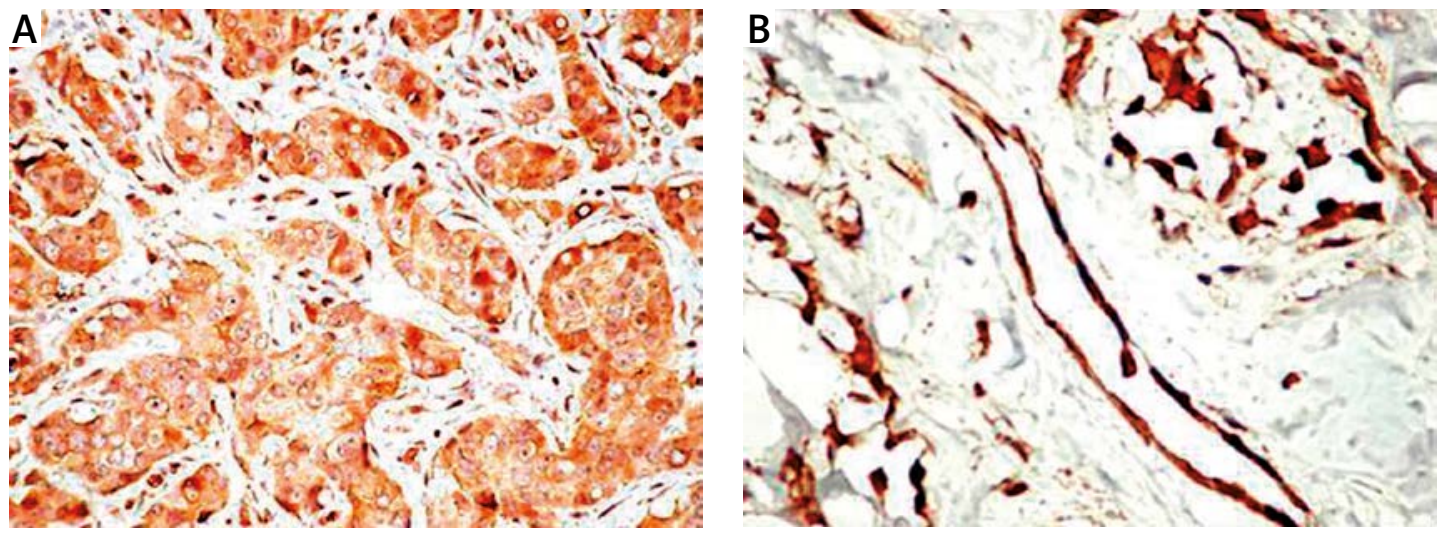

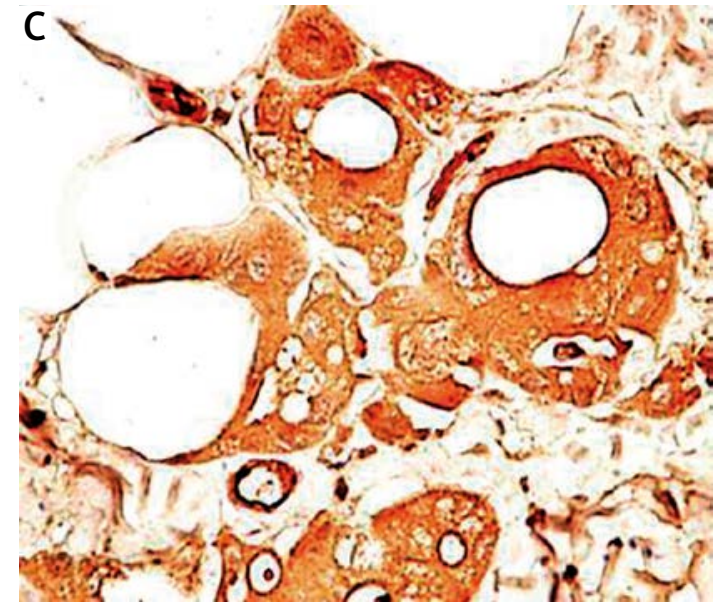

been demonstrated that endothelial migration is PDGFR $\alpha$ dependent signaling [56]. It seems that PDGF family members are not long-term chemotactic factors for ECs, but are mandatory for upstream regulation in order to activate or induce the secretion of different signaling proteins [57]. Recently, it was demonstrated that PDGFR $\beta$ induced signaling in the vasculature may determine different types of transcriptional responses. Different members of the PDGF family are linked to a series of transcriptional targets under different circumstances. Based on these remarks, the specific cellular responses that occur after PDGF action can be explained.

\section{Normal versus pathological}

Despite the fact that the same signaling molecule is implicated, the expression, function and the PDGF mechanism of action largely vary in normal compared to pathological conditions. This differential expression concerns the receptors associated with this family as well. Although lymphangiogenesis (normal and pathological) as well as the behavior of the PDGF are aspects about which very little is known or understood, a parallel can be made between the normal and the tumor tissue regarding the PDGF action.
Figure 2. PDGFR $\alpha$ expression in primary breast cancer cells (A), peritumoral lymphatic vessels (B) and corresponding nodal metastasis (C)

There are two main theories regarding lymphatic vessel development: the first stating that the conversion of the venous EC into a lymphatic one by Prox-1 induction [58] and the second starting from lymphangioblasts.

The main factor involved in normal angiogenesis and lymphangiogenesis induction is the vascular endothelial growth factor (VEGF) [59, 60], PDGF being just a factor that participates in this process. The VEGF induces migration, proliferation and cell growth followed by the future development of blood or lymphatic vessels [61].

In normal conditions, PDGF acts in a predominant paracrine manner in order to encourage angiogenesis and lymphangiogenesis.

In the case of all tumors, the lymphatic vessel profile is changed. This fact has been demonstrated in numerous studies based on experimental models. Most of these studies target the functional implications of VEGF and VEGFR in pathological angiogenesis (mostly) and lymphangiogenesis [62], the implications of PDGF and PDGFR being recently discovered.

Within tumors, high levels of PDGF (especially homodimer B), as well as the overexpression of PDGFR $\beta$, are detected mainly in the peripheral lymphatic vessels. The newly formed pathological lymphatic vessels, unlike the physiological 
ones, present a diameter above the average, being tortuous vessels with a disorganized layout. The PDGF-BB and PDGF-AA (as well as PDGF-CC) are capable of inducing tumor lymphangiogenesis, but while homodimer B induces the development of a large number of lymphatic vessels, homodimer A has lower activity. In the case of angiogenesis, there are no significant differences between the two members of the family. Within the tumor, the presence of lymphatic vessels with the lack of normal structure can be observed: the lack of the typical lymphatic vessel structure, an element that is considered by many authors as an important basis of tumor cell dissemination. To generate pathological lymphatic vessels, cells secrete VEGF-C, which can activate the pro-lymphangiogenic role of PDGF-BB. Thus, considering the tumor type, a variability of structure and function of growth factors appears.

As in the case of the normal tissue, the additive and synergic action of growth factors has been demonstrated. From this point of view, the most efficient therapeutic means of stopping tumor growth and expansion would be the administration of both PDGFR inhibitors and VEGFR antagonists $[63,64]$. Such a therapeutic means would however have negative effects for the organism because of its high toxicity.

As observe in many types of tumors, the PDGF action is predominantly autocrine [65-68], unlike the paracrine action that appears in normal conditions.

What is the reason behind this modification of the means of action? Is it possible to represent the cause by the aberrant secretion of PDGF?

Under the action of growth factors, the pathological lymphatic system is insufficient and atypical, and unlike the normal one it does not present the ability of lymphatic drainage because of the blockage of the lumen by tumor cells.

The common characteristics for normal and pathological lymphangiogenesis are on one hand the fact that in both situations the action of a signaling molecule is needed, while on the other hand the lymphatic vessel phenotype is changed according to the conditions of the microenvironment. Thus, lymphatic vessels are heterogeneous structures. It is possible that the phenotype variability is associated with functional and structural variability of growth factors. The possibility of certain members expressed only at some point remain a hypothesis. In pathological conditions, it is extremely difficult to use efficient therapeutic targets because of the variability phenomenon.

These could be some of the major differences appearing between the normal and the pathological lymphangiogenesis. The PDGF variability appears not only in several tumor types but also in normal conditions in different organs.

\section{Why are there different expressions? Why variability?}

Why are there different expressions? There is no certain answer to this question. There are numerous controversies regarding the expression of different members in certain cell types in normal conditions but also within common tumors.

One possible answer could be the development of different stromal components from various normal tissues with a high variability in the cell types able to secrete PDGFs. Also the autocrine and paracrine mechanism of action could be specific for each normal and tumor microenvironment. But all these hypotheses need to be validated by future studies.

The PDGF members are expressed in various cell types in physiological conditions. It seems that the strongest expression for PDGF-A was identified in the heart, the skeletal muscle and in the pancreas, while the BB dimer presents the highest expression in the heart and placenta, and moderate levels in other organs.

Within the PDGF family, only form A and form $B$ are capable of forming the one heterodimer that has been evidenced so far: PDGF-AB. Even PDGF-C presents high expression in the heart, the kidney, the adrenal gland and the pancreas, but lower levels have been evidenced in the liver and the ovary. The highest expression of D form appears in the pancreas, ovaries, and heart, while their expression in the brain, lungs, and skeletal muscles has not been documented [4]. Also, the expression of PDGF-C is absent in the spleen and the colon [14]. The differential expression has been identified not only in normal cells but also in tumor cell lines [69].

The literature reveals that different PDGF members are expressed within the same organ, but they may appear with a different structure. The structural variety is in this case associated with a functional one.

Is it possible for structural differences to appear within the same PDGF form? Supposing that this might be possible, the basis of this phenomenon may be represented by a mutation that appears in the gene encoding the synthesis of the PDGF form.

Not only the growth factor itself presents differential expression but also the growth factor associated receptor. Thus, PDGFR $\alpha$ is expressed by tumor cells in glioblastomas and PDGFR $\beta$ is expressed by the ECs in the newly formed blood vessels [69]. In the transplanted tumor models, the number and the density of perivascular cells are much higher, because of cancerous cells overexpressing PDGF-B. It seems that perivascular cells are capable of responding to ectopic sources of PDGF-B through the migration phenomenon from the ECs. Regarding this aspect, PDGF-B is not 
only a mitogen factor for perivascular cell precursors but at the same time it guides the migration during tumor angiogenesis.

In lymphangiogenesis, intra- and peritumoural lymphatic vessels are key elements that facilitate tumor cell spreading in regional lymph nodes. This fact has been evidenced in numerous common types of cancer such as breast cancer, colorectal carcinoma and malignant melanoma. Amongst the PDGF family members, PDGF-B expression has been evidenced in breast cancer. It seems that dimer B of PDGFs is a chemotactic factor for primary LECs. The effect of PDGF in perivascular cells and in tumor stroma points out the possibility that PDGF acts along with other angiogenic molecules in order to sustain both angiogenesis and lymphangiogenesis.

Through stopping PDGFR activity, the decrease of cell proliferation may be determined, and the increase of apoptosis rate may be induced because the high levels of PDGF and PDGFR are often associated with a bad prognosis and a low response to chemotherapy. Due to the fact that tumor cells and tumor associated ECs express PDGFRs, the receptor stopping pathway is based on the use of a PDGFR-tyrosine kinase, in order to block the PDGFR phosphorylating domain.

\section{Upcoming challenges!}

Besides this lymphangiogenic growth factor with direct action, the other factors intervening in lymphangiogenesis are fibroblastic growth factor 2, angiopoietin 2, neuropilin 2 and Prox-1. Is it possible for PDGF action to resemble that of VEGF during embryogenesis in the context of lymphatic vessel formation? Is it possible to derive two types of polypeptides with similar action? Despite all this, it seems that ECs do not express the $\beta$ receptor in physiological conditions. Is it possible, under some specific circumstances, for the VEGF to replace the action of the PDGF, considering the fact that the latter has a higher lymphangiogenic action compared to that of PDGF? Is it possible to neglect the PDGF action in embryonic lymphangiogenesis opposed to that of VEGF? It may be so.

In the end, almost all experimental studies on the functional implications of the PDGF are mostly based on pathological/tumor lymphangiogenesis, its pro-lymphangiogenic action, in normal conditions but mentioned along with that of VEGF. Despite all this, the action of the VEGF in pathological conditions should not be neglected. The capacity of tumor cells to secrete VEGF-C in order to induce lymphatic vessel formation is well known, the next step being represented by the dissemination towards the adjacent lymph nodes. Is it possible to delay PDGF interventions in normal lymphangiogenesis compared to that of VEGF? Phenotypic modification of lymphatic vessels through the intervention of microenvironmental factors is well known. Changing phenotype, in normal and pathological conditions, is in need of the interference of other isoforms of the PDGF family, apart from those known. If the PDGF family has members with antibody-like domains, is it possible for PDGF members to possess a similar capacity of isotype switching? Antibody isotype switching confers on them the capacity to interact with a large range of antigens. The isotype switching phenomenon is closely related to the gene that encodes the antibody chain and confers their great capacity to form about 10 types in order to interact with the antigens. Is it possible that because of the great phenotype variety those lymphatic vessels can present, there is a great variety of growth factors? (not only regarding the PDGF family).

Under the circumstances in which this phenomenon could be possible, in pathological conditions, it is very difficult to find characteristic inhibitors of great specificity for each type of growth factor generated.

\section{Acknowledgments}

This work was supported by UEFISCDI grants IDEI No. 345/2011, and No. TE109/2011 of the Romanian Ministry of Education and Research. Also, the present work was supported by Internal Grant from "Victor Babes" University of Medicine and Pharmacy Timisoara Romania entitled Targeted prognostic and therapeutic value of molecular profile of head and neck premalignant lesions and squamous cell carcinomas /2014,- Innovative Basic Research Program PIII-C1-CFI-2014/2015-02.

\section{Conflict of interest}

The authors declare no conflict of interest.

\section{References}

1. Betsholtz C, Karlsson L, Lindahl P. Developmental roles of platelet derived growth factors. Bioessays 2001; 23: 494-507.

2. Chen PH, Chen X, He X. Platelet-derived growth factors and their receptors: structural and functional perspectives. Biochim Biophys Acta 2013; 1834: 2176-86.

3. Andrade J, Gallini R, Betsholtz C. Role of platelet-derived growth factors in physiology and medicine. Genes Dev 2008; 22: 1276-312.

4. Fredriksson L, Li H, Eriksson U. The PDGF family: four gene products form five dimeric isoforms. Cytokine Growth Factor Rev 2004; 15: 197-204.

5. Reigstad LJ, Varhaug JE, Lillehaug JR. Structural and functional specificities of PDGF-C and PDGF-D, the novel members of the platelet-derived growth factors family. FEBS J 2005; 272: 5723-41.

6. Bergsten E, Uutela M, Li X, et al. UPDGF-D is a specific and protease-activated ligand for the PDGF $\mathrm{b}$ receptor. Nature Cell Biol 2001; 3: 512-6. 
7. Oefner C, D’Arcy A, Winkler FK, Eggimann B, Hosang M. Crystal structure of human platelet-derived growth factor BB. EMBO J 1992; 11: 3921-6.

8. Li X, Ponten A, Aase K, et al. PDGF-C is a new protease-activated ligand for the PDGF alpha-receptor. $\mathrm{Na}$ ture Cell Biol 2000; 2: 302-9.

9. Heldin $\mathrm{CH}$, Ostman A, Rönnstrand L. Signal transduction via the Platelet-derived growth factor receptors. Biochim Biophys Acta 1998; 1378: F79-113.

10. Wennstroèm $S$, Siegbahn A, Yokote $K$, et al. Membrane ruffling and chemotaxis transduced by the PDGF beta-receptor require the binding site for phosphatidylinositol 3' kinase. Oncogene 1994; 9: 651-60.

11. Fambrough D, McClure K, Kazlaskas A, Lander E. Diverse signalling pathways activated by growth factor receptors induce broadly overlapping rather than independent sets of genes. Cell 1999; 97: 727-41.

12. Rosenkranz S, Kazlauskas A. Evidence for distinct signaling properties and biological responses induced by the PDGF receptor-alpha and beta subtypes. Growth Factors 1999; 16: 201-16.

13. Heldin $\mathrm{CH}$, Westermark B. Mechanism of action and in vivo role of platelet derived growth factor. Physiol Rev 1999; 79: 1283-316.

14. Raica M, Cimpean AM. Platelet-derived growth factor (PDGF)/PDGF receptors(PDGFR) axis as target for antitumor and antiangiogenic therapy. Pharmaceuticals 2010; 3: 572-99.

15. Hellstroèm $M$, Kaleân $M$, Lindahl $P$, Abramsson $A$, Betsholtz C. Role of PDGF-B and PDGFR-beta in recruitment of vascular smooth muscle cells and pericytes during embryonic blood vessel formation in the mouse. Development 1999; 126: 3047-55.

16. Williams SP, Karnezis T, Achen MG, Stacker SA. Targeting lymphatic vessel functions through tyrosine kinases. J Angiogenes Res 2010; 2: 13.

17. Ehnman M, Missiaglia E, Folestad E, Selfe J, et al. Distinct effects of ligand-induced PDGFRalpha and PDGFRbeta signaling in the human rhabdomyosarcoma tumor cell and stroma cell compartments. Cancer Res 2013; 73: 2139-49.

18. Schoppmann SF, Alidzanovic L, Schultheis A, Perkmann T, Brostjan C, Birner P. Thrombocytes correlate with lymphangiogenesis in human esophageal cancer and mediate growth of lymphatic endothelial cells in vitro. PLoS One 2013; 8: e66941.

19. Linares PM, Gisbert JP. Role of growth factors in the development of lymphangiogenesis driven by inflammatory bowel disease: a review. Inflamm Bowel Dis 2011; 17: 1814-21.

20. Reigstad LJ, Sande HM, Fluge O, et al. Platelet-derived growth factor (PDGF) -C, a PDGF family member with a vascular endothelial growth factor-like structure. J Biol Chem 2003; 278: 17114-20.

21. Achen MG, Stacker SA. Tumor lymphangiogenesis and metastatic spread - new players begin to emerge. Int J Cancer 2006; 119: 1755-60.

22. Onimaru $M$, Yonemitsu Y, Fujii T, et al. VEGF-C regulates lymphangiogenesis and capillary stability by regulation of PDGF-B. Am J Physiol Heart Circ Physiol 2009; 297: H1685-96.

23. Swoboda A, Schanab O, Tauber S, et al. MET expression in melanoma correlates with a lymphangiogenic phenotype. Hum Mol Genet 2012; 21: 3387-96.

24. Onimaru M, Yonemitsu Y. Angiogenic and lymphangiogenic cascades in the tumor microenvironment. Front Biosci (Schol Ed) 2011; 3: 216-25.
25. Pfister C, Pfrommer H, Tatagiba MS, Roser F. Vascular endothelial growth factor signals through platelet-derived growth factor receptor beta in meningiomas in vitro. Br J Cancer 2012; 107: 1702-13.

26. Suzuki S, Dobashi Y, Hatakeyama Y, et al. Clinicopathological significance of platelet-derived growth factor (PDGF)-B and vascular endothelial growth factor-A expression, PDGF receptor-beta phosphorylation, and microvessel density in gastric cancer. BMC Cancer 2010; 10: 659 .

27. Engelmann D, Mayoli-Nüssle D, Mayrhofer C, et al. E2F1 promotes angiogenesis through the VEGF-C/VEGFR-3 axis in a feedback loop for cooperative induction of PDGF-B. J Mol Cell Biol 2013; 5: 391-403.

28. Schimanski CC, Schlaegel F, Jordan M, et al. VEGF-D correlates with metastatic disease in gastric cancer patients undergoing surgery. World J Surg 2011; 35: 1010-6.

29. Cao R, Björndahl MA, Religa P, et al. PDGF-BB induces intratumoral lymphangiogenesis and promotes lymphatic metastasis. Cancer Cell 2004; 6: 333-45.

30. van den Akker NM, Winkel LC, Nisancioglu MH, et al. PDGF-B signaling is important A for murine cardiac development: its role in developing atrioventricular valves, coronaries, and cardiac innervation. Dev Dyn 2008; 237: 494-503.

31. Petrova TV, Mäkinen T, Mäkelä TP, et al. Lymphatic endothelial reprogramming of vascular endothelial cells by the Prox-1 homeobox transcription factor. EMBO J 2002; 21: 4593-9.

32. Hong YK, Harvey N, Noh YH, et al. Prox1 is a master control gene in the program specifying lymphatic endothelial cell fate. Dev Dyn 2002; 225: 351-7.

33. Wigle JT, Oliver G. Prox 1 function is required for the development of the murine lymphatic system. Cell 1999; 98: 769-78

34. Donnem T, Al-Saad S, Al-Shibli K, Busund LT, Bremnes RM. Co-expression of PDGF-B and VEGFR-3 strongly correlates with lymph node metastasis and poor survival in non-small-cell lung cancer. Ann Onco 2010; 21: 223-31.

35. Terada T. Protein expression and gene mutation status of KIT and PDGFRA in renal cell carcinoma. Heston Histopathol 2012; 27: 297-302.

36. Ghanem M, Nijman R, Safan M, van der Kwast T, Vansteenbrugge $G$. Expression and prognostic value of platelet-derived growth factor-AA and its receptor alpha in nephroblastoma. BJU Int 2010; 106: 1389-93.

37. Knösel T, Chen Y, Altendorf-Hofmann A, et al. High KIT and PDGFRA are associated with shorter patients survival in gastroenteropancreatic neuroendocrine tumors, but mutations are a rare event. I Cancer Res Clin Oncol 2012; 138: 397-403.

38. Malavaki CJ, Roussidis AE, Gialeli C, et al. Imatinib as a key inhibitor of the platelet-derived growth factor receptor mediated expression of cell surface heparan sulfate proteoglycans and functional properties of breast cancer cells. FEBS J 2013; 280: 2477-89.

39. Russell MR, Liu Q, Fatatis A. Targeting the \{alpha\} receptor for platelet-derived growth factor as a primary or combination therapy in a preclinical model of prostate cancer skeletal metastasis. Clin Cancer Res 2010; 16: 5002-10.

40. Liu Q, Jernigan D, Zhang Y, Fatatis A. Implication of platelet-derived growth factor receptor alpha in prostate cancer skeletal metastasis. Chin J Cancer 2011; 30: 612-9.

41. Ding W, Knox TR, Tschumper RC, et al. Platelet-derived growth factor (PDGF)-PDGF receptor interaction activates bone marrow-derived mesenchymal stromal 
cells derived from chronic lymphocytic leukemia: implications for an angiogenic switch. Blood 2010; 116: 2984-93.

42. Jain RK, Lahdenranta J, Fukumura D. Targeting PDGF signaling in carcinoma-associated fibroblasts controls cervical cancer in mouse model. PLoS Med 2008; 5: e24.

43. Kodama M, Kitadai $Y$, Sumida T, et al. Expression of platelet-derived growth factor (PDGF)-B and PDGF- receptor beta is associated with lymphatic metastasis in human gastric carcinoma. Cancer Sci 2010; 101: 1984-9.

44. Inoue A, Moriya H, Katada N, et al. Intratumoral lymphangiogenesis of esophageal cell carcinoma and relationship with regulatory factors and prognosis. Pathol Int 2008; 58: 611-9.

45. Liu P, Zhou J, Zhu H, et al. VEGF-C promotes the development of esophageal cancer via regulating CNTN-1 expression. Cytokine 2011; 55: 8-17.

46. Martinez LM, Vallone VB, Labovsky V, et al. Changes in the peripheral blood and bone marrow from untreated advanced breast cancerpatients that are associated with the establishment of bone metastases. Clin Exp Metastasis 2014; 31: 213-32.

47. Frings $O$, Augsten $M$, Tobin NP, et al. Prognostic significance in breast cancer of a gene signature capturing stromal PDGF signaling. Am J Pathol 2013; 182: 2037-47.

48. Liu DS, Zhang XJ, Chen G, Lu CF, Dong HY. Expression of platelet-derived growth factor receptor alpha and its ligand PDGF-A in breast cancer. Xi Bao Yu Fen Zi Mian Yi Xue Za Zhi 2011; 27: 313-6.

49. Hiraga T, Nakamura H. Imatinib mesylate suppresses bone metastases of breast cancer by inhibiting osteoclasts through the blockade of c-Fms signals. Int J Cancer 2009; 124: 215-22.

50. Liu J, Liao S, Huang Y, et al. PDGF-D improves drug delivery and efficacy via vascular normalization, but promotes lymphatic metastasis by activating CXCR4 in breast cancer. Clin Cancer Res 2011; 17: 3638-48.

51. Ahmad A, Wang Z, Kong D, et al. Platelet-derived growth factor-D contributes to aggressiveness of breast cancer cells by up-regulating Notch and NF-kappaB signaling pathways. Breast Cancer Res Treat 2011; 126: 15-25.

52. Schito L, Rey S, Tafani M, et al. Hypoxia-inducible factor 1-dependent expression of platelet-derived growth factor B promotes lymphatic metastasis of hypoxic breast cancer cells. Proc Natl Acad Sci USA 2012; 109: E2707-16.

53. Semenza GL. Cancer-stromal cell interactions mediated by hypoxia-inducible factors promote angiogenesis, lymphangiogenesis, and metastasis. Oncogene 2013; 32: 4057-63.

54. Yokoyama Y, Mori S, Hamada Y, et al. Platelet derived growth factor regulates breast cancer progression via beta-catenin expression. Pathobiology 2011; 78: 253-60.

55. Brennan J, Tilmann C, Capel B. PDGFR-alpha mediates testis cord organization and fetal Leydig cell development in the XY gonad. Genes Dev 2003; 17: 800-10.

56. Hoch RV, Soriano P. Roles of PDGF in animal development. Development 2003; 130: 4769-84.

57. Alitalo K, Carmeliet P. Molecular mechanisms of lymphangiogenesis in health and disease. Cancer Cell 2002; 1: 219-27.

58. Ferrara N. Molecular and biological properties of vascular endothelial growth factor. J Mol Med 1999; 77: 527-43.
59. Ferrara N. Role of vascular endothelial growth factor in regulation of physiological angiogenesis. Am J Physiol Cell Physiol 2001; 280: 1358-66.

60. Adams RH, Alitalo K. Molecular regulation of angiogenesis and lymphangiogenesis. Nat Rev Mol Cell Biol 2007; 8: 464-78.

61. Kieran MW, Kalluri R, Cho YJ. The VEGF pathway in cancer and disease: responses, resistance, and the path forward. Cold Spring Harb Perspect Med 2012; 2: a006593.

62. Laschke MW, Elitzsch A, Vollmer B, Vajkoczy P, Menger MD. Combined inhibition of vascular endothelial growth factor (VEGF), fibroblast growth factor and platelet-derived growth factor, but not inhibition of VEGF alone, effectively suppress angiogenesis and vessel maturation in endometriotic lesions. Hum Reprod 2006; 21: 262-8.

63. Saharinen P, Alitalo K. Double target for tumor mass destruction. J Clin Invest 2003; 111: 1277-80.

64. Őstman A. PDGF receptors-mediators of autocrine tumor growth and regulators of tumor vasculature and stroma. Cytokine Growth Factor Rev 2004; 15: 275-86.

65. Krasagakis K, Garbe C, Orfanos CE. Cytokines in human melanoma cells: synthesis, autocrine stimulation and regulatory functions - an overview. Melanoma Res 1993; 3: 425-33.

66. Lokker NA, Sullivan CM, Hollenbach SJ, Israel MA, Giese NA. Plateletderived growth factor (PDGF) autocrine signaling regulates survival and mitogenic pathways in glioblastoma cells: evidence that the novel PDGF-C and PDGF-D ligands may play a role in the development of brain tumors. Cancer Res 2002; 62: 3729-35.

67. Hermanson M, Funa K, Hartman M, et al. Platelet-derived growth factor and its receptors in human glioma tissue: expression of messenger RNA and protein suggests the presence of autocrine and paracrine loops. Cancer Res 1992; 52: 3213-9.

68. Nister M, Claesson-Welsh L, Eriksson A, Heldin CH, Westermark B. Differential expression of platelet-derived growth factor receptors in human malignant glioma cell lines. J Biol Chem 1991; 266: 16755-63.

69. Lokker NA, O'Hare JP, Barsoumian A, et al. Functional importance of platelet derived growth factor (PDGF) receptor extracellular immunoglobulin-like domains Identification of PDGF binding sites and neutralizing monoclonal antibodies. J Biol Chem 1997; 272: 33037-44. 